\title{
Macroscopic effects of the spectral structure in turbulent flows
}

\author{
Tuan Tran ${ }^{1}$, Pinaki Chakraborty ${ }^{2 \star}$, Nicholas Guttenberg ${ }^{3}$, Alisia Prescott ${ }^{4}$, Hamid Kellay ${ }^{5}$, \\ Walter Goldburg ${ }^{4}$, Nigel Goldenfeld ${ }^{3 \dagger}$ and Gustavo Gioia ${ }^{1 \dagger}$
}

\begin{abstract}
There is a missing link between the macroscopic properties of turbulent flows ${ }^{1-4}$, such as the frictional drag $^{5}$ of a wallbounded flow, and the turbulent spectrum ${ }^{1,6,7}$. The turbulent spectrum is a power law of exponent $\alpha$ (the 'spectral exponent') that gives the characteristic velocity of a turbulent fluctuation (or 'eddy') of size $s$ as a function of $s$ (ref. 1). Here we seek the missing link by comparing the frictional drag in soap-film flows ${ }^{8}$, where $\alpha=3$ (refs 9,10), and in pipe flows ${ }^{5}$, where $\alpha=5 / 3$ (refs 11,12). For moderate values of the Reynolds number $\mathrm{Re}$, we find experimentally that in soap-film flows the frictional drag scales as $\operatorname{Re}^{-1 / 2}$, whereas in pipe flows the frictional drag scales ${ }^{13}$ as $\operatorname{Re}^{-1 / 4}$. Each of these scalings may be predicted from the attendant value of $\alpha$ by using a new theory ${ }^{14-16}$, in which the frictional drag is explicitly linked to the turbulent spectrum.
\end{abstract}

Turbulent flows past a wall experience frictional drag, the macroscopic property of a flow that sets the cost of pumping oil through a pipeline, the draining capacity of a river in flood, and other quantities of engineering interest ${ }^{2,3,5,17,18}$. The frictional drag is defined as the dimensionless ratio $f=\tau / \rho U^{2}$, where $\tau$ is the shear stress or force per unit area that develops between the flow and the wall, $\rho$ is the density of the fluid and $U$ is the mean velocity of the flow. Already in eighteenth-century France, $f$ was the subject of large-scale experiments carried out in connection with the design of a waterworks for the city of Paris ${ }^{19,20}$. In 1883 it was predicted ${ }^{21}$, and subsequently confirmed by numerous experiments, that in pipe flows $f$ depends on the Reynolds number $\operatorname{Re}=U d / v$, where $d$ is the diameter of the pipe and $v$ is the kinematic viscosity of the fluid. For pipe flows of moderate turbulent strength (starting from $\operatorname{Re} \approx 2,500$ and up to $\operatorname{Re} \approx 100,000$ ) the experimental results are well described $^{13}$ by the Blasius empirical scaling, $f \propto \mathrm{Re}^{-1 / 4}$. (Throughout this letter, the symbol ' $\propto$ ' may be changed to the symbol ' $=$ ' by introducing a dimensionless proportionality factor, for example, $f=C \operatorname{Re}^{-1 / 4}$.) The celebrated theory ${ }^{3,5}$ of the frictional drag was formulated 80 years ago by Ludwig Prandtl, the founder of turbulent hydraulics, and numerous variants ${ }^{5,13,22}$ and alternatives ${ }^{23,24}$ of Prandtl's theory have since been proposed. Although Prandtl's theory and its variants and alternatives yield disparate mathematical expressions for $f$ as a function of $\mathrm{Re}$, for moderate values of Re they all give predictions in good numerical accord with the Blasius empirical scaling. Yet these theories have been predicated on dimensional analysis and similarity assumptions, without reference to the spectral structure of the turbulent fluctuations. As a result, these theories cannot be used to reveal the missing link between the frictional drag and the turbulent spectrum.
The turbulent spectrum is a function of the wavenumber $k, E(k)$. The physical significance of $E(k)$ can be grasped from the expression ${ }^{3} \quad u_{s} \propto\left(\int_{1 / s}^{\infty} E(k) \mathrm{d} k\right)^{1 / 2}$, which gives the characteristic velocity $u_{s}$ of a turbulent eddy of size $s$ in the flow. For the spectrum of the spectral exponent $\alpha$ we can write ${ }^{16}$ $E(k) \propto U^{2} L^{(1-\alpha)} k^{-\alpha}$, and therefore

$$
u_{s} \propto U(s / L)^{(\alpha-1) / 2}
$$

where $U$ is the mean velocity of the flow and $L$ is a characteristic length. A single type of spectrum is possible in three-dimensional (3D) flows: the 'energy cascade,' for which $\alpha=5 / 3$ (ref. 12). Thus, for example, in turbulent pipe flows the spectral exponent is $5 / 3$ and $L=d$, the diameter of the pipe. Two-dimensional (2D) turbulence (a type of turbulence that may be realized in a soap film) differs from $3 \mathrm{D}$ turbulence in several crucial respects, most notably in that in two dimensions there is no vortex stretching. As a result, a different type of spectrum is possible in 2D flows: the 'enstrophy cascade', for which $\alpha=3$ (refs 9,10). Thus, for example, in turbulent soap-film flows the spectral exponent is 3 and $L=w$, the width of the soap film.

To study soap-film flows we hang a soap film between two long, vertical, mutually parallel wires a few centimetres apart from one another (Fig. 1a). Driven by gravity, a steady vertical flow soon becomes established within the film. In this case, the thickness $h$ of the film is roughly uniform on any cross-section of the film, typically $h \approx 10 \mu \mathrm{m}$, much smaller than the width $w$ and the length of the film (Fig. 1a). As a result, the velocity of the flow lies on the plane of the film, and the flow is 2D.

We make the flow turbulent by piercing the film with a comb, as indicated in Fig. 1a, so that the flow is stirred as it moves past the teeth of the comb. To visualize the flow, we cast monochromatic light on a face of a film and observe the interference fringes that form there. These fringes (Fig. 1b) reflect small changes in the local thickness of the film. (The thickness is constant along a fringe; it differs by a fraction of the wavelength of the light, or a fraction of a micrometre, between any two successive fringes.) The small changes in thickness in turn reflect small changes in the absolute value of the instantaneous velocity of the flow ${ }^{8}$. Thus, Fig. 1b may be interpreted as a map of the instantaneous spatial distribution of turbulent fluctuations downstream of the comb.

We compute the spectrum $E(k)$ at numerous points on the film from measurements carried out with a laser Doppler velocimeter (LDV; see the Methods section). In Fig. 1c we show a few typical $\log -\log$ plots of $E$ versus $k$. The slope of these plots represents

\footnotetext{
${ }^{1}$ Department of Mechanical Science and Engineering, University of Illinois, Urbana, Illinois 61801, USA, ${ }^{2}$ Department of Geology, University of Illinois, Urbana, Illinois 61801, USA, ${ }^{3}$ Department of Physics, University of Illinois, Urbana, Illinois 61801, USA, ${ }^{4}$ Department of Physics and Astronomy, University of Pittsburgh, Pittsburgh, Pennsylvania 15260, USA, ${ }^{5}$ Centre de Physique Moléculaire Optique et Hertzienne (UMR 5798 CNRS), Université Bordeaux I, 33405 Talence, France. ${ }^{\dagger}$ These authors contributed equally to this work. ${ }^{*}$ e-mail: chakrabo@illinois.edu.
} 
a

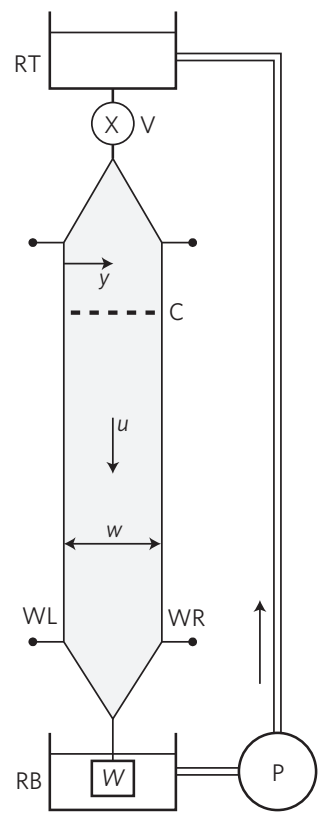

c

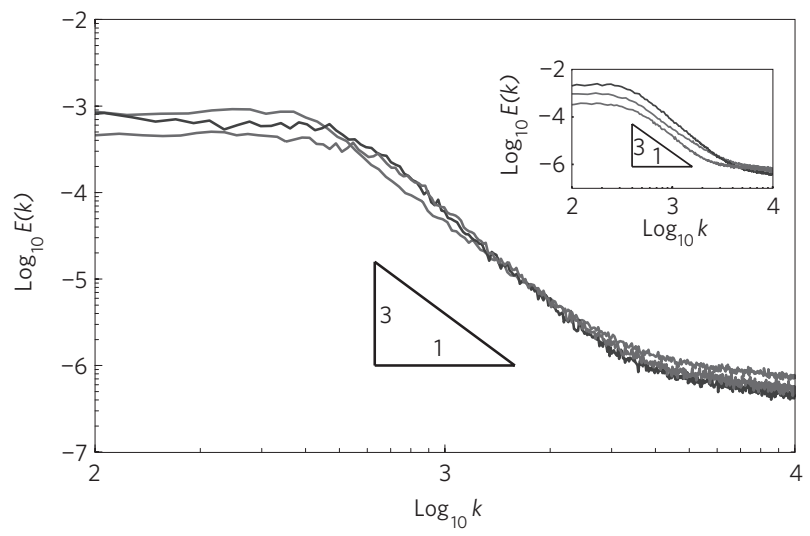

Figure 1 | Experimental set-up used to study steady, gravity-driven, 2D soap-film flows. a, Wires WL and WR are thin nylon wires (diameter $=0.5 \mathrm{~mm}$ ) kept taut by weight $W$. The film hangs from the wires; its width increases from 0 to $w$ over an expansion section, then remains constant and equal to $w$ over a section of length $\approx 1 \mathrm{~m}$. Reservoir $\mathrm{RT}$ contains a soapy solution (2.5\% Dawn Nonultra in water; $v=0.01 \mathrm{~cm}^{2} \mathrm{~s}^{-1}$ ), which flows through valve $V$ and into the film. After flowing through the film, the soapy solution drains into reservoir $\mathrm{RB}$ and returns to reservoir $\mathrm{RT}$ through pump $\mathrm{P}$. Turbulence is generated by comb $\mathrm{C}$, of tooth diameter $\approx 0.5 \mathrm{~mm}$ and tooth spacing $\approx 2 \mathrm{~mm}$. We carry out all measurements at a distance of at least $10 \mathrm{~cm}$ downstream of the comb. Axis $y(0 \leq y \leq w)$ has its origin at wire $\mathrm{WL}$, as indicated. $\mathbf{b}$, Interference fringes in yellow light (wavelength $=0.589 \mu \mathrm{m}$ ) make it possible to visualize the generation of $2 \mathrm{D}$ turbulence from the comb. c, Typical log-log plots of the spectrum E(k), from LDV measurements carried out on points of the film close to one of the wires and (inset) along the centreline of the flow.

the spectral exponent $\alpha$; in our experiments the slope is slightly larger than 3 , consistent with previous experiments with soapfilm flows ${ }^{8,25}$ and close to the theoretical value of $\alpha$ for the enstrophy cascade $(\alpha=3)$.

By using the same LDV we measure the mean (time-averaged) velocity $u$ at any point on the film (see the Methods section). Successive measurements of $u$ along a cross-section of the film give the 'mean velocity profile' $u(y)$ of that cross-section. In Fig. 2 a we show a few typical plots of $u(y)$ over the entire width of the film (that is, from wire to wire, or for $0 \leq y \leq w)$. From a mean velocity profile we compute the mean velocity of the flow as $U=(1 / w) \int_{0}^{w} u(y) \mathrm{d} y$, and the Reynolds number of the flow as $\operatorname{Re}=U w / v$.

In Fig. $2 \mathrm{~b}$ we show a few typical plots of $u(y)$ close to one of the wires, where $u$ depends linearly on $y$ on a narrow (about $0.2 \mathrm{~mm}$ ) viscous layer. We have verified that the Reynolds shear stress vanishes in the viscous layer (Fig. 2c,d). We have also verified that the thickness of the film is nearly uniform in the viscous layer (Fig. 2e,f).

From the slope $G$ of a mean velocity profile in the viscous layer (for example, Fig. 2b), we compute the shear stress between the flow and the wire as $\tau=\rho \nu G$. The frictional drag follows from the definition, $f=\tau / \rho U^{2}$, as $f=v G / U^{2}$.

An apparent slip velocity $U_{S}$ is conspicuous in the plots of Fig. $2 b$, and is likely to represent 3D and surface-tension effects associated with the complex flow at the contact between a film and a wire. The value of $U_{\mathrm{S}}$ tends to lessen where we use thinner wires or brand new wires. By using a variety of wires, we have been able to realize several flows with the same value of Re but widely differing values of $U_{\mathrm{S}}$. We have verified that the frictional drag of these flows is the same within experimental error (for example, Fig. 3), in spite of the widely differing values of $U_{S}$. We conclude that the frictional drag does not depend on the apparent slip velocity (except perhaps through the Reynolds number).

In Fig. 4 we show a log-log plot of $f$ versus Re. The plot consists of five sets of data points from numerous turbulent soap-film flows; four sets were taken at Pittsburgh, and one at Bordeaux in an independent experimental set-up. The cloud of data points is consistent with the scaling, $f \propto \mathrm{Re}^{-1 / 2}$, and inconsistent with the Blasius empirical scaling, $f \propto \mathrm{Re}^{-1 / 4}$, which is known to prevail in turbulent pipe flows.

Our experimental results may be explained using a recently proposed theory of the frictional $\mathrm{drag}^{14-16}$. In this theory, the frictional drag is produced by turbulent eddies that transfer momentum between the wall or wire (where the fluid carries a negligible momentum per unit mass) and the turbulent flow (where the fluid carries a sizable momentum per unit mass). The theory is applicable to flows on rough walls, but where the walls are smooth (as in our experiments), the theory predicts that $f \propto u_{\eta} / U$ (refs $14-16,22,26-29)$. Here $\eta$ is the size, and $u_{\eta}$ the characteristic velocity, of those eddies with an intrinsic Reynolds number of 1 , so that $u_{\eta} \eta / \nu=1$. By setting $s=\eta$ in (1) and combining the result with $u_{\eta} \eta / v=1$ and $\operatorname{Re}=U L / v$, we conclude that if the energy spectrum of the flow has the spectral exponent $\alpha$, then the scaling, $u_{\eta} \propto U \operatorname{Re}^{(1-\alpha) /(1+\alpha)}$, must hold. It follows that

$$
f \propto \operatorname{Re}^{(1-\alpha) /(1+\alpha)}
$$

and the functional dependence of the frictional drag on the Reynolds number is set by the spectral exponent $\alpha$. For pipe flows $\alpha=5 / 3$, and (2) yields ${ }^{15} f \propto \mathrm{Re}^{-1 / 4}$, consistent with the Blasius scaling. In contrast, for soap-film flows $\alpha=3$, and (2) yields ${ }^{16} f \propto \mathrm{Re}^{-1 / 2}$, consistent with our experimental results (Fig. 4).

From our experiments with 2D soap-film flows we infer that the long-standing and widely accepted theory ${ }^{5}$ of the frictional drag between a turbulent flow and a wall is incomplete. This classical theory does not take into account the structure of the turbulent fluctuations, and cannot distinguish between 2D and 3D turbulent flows. Our data on soap-film flows, as well as the available data on pipe flows, are, however, consistent with the predictions of a recently proposed theory of the frictional $\mathrm{drag}^{14-16}$. This new theory perforce relates the frictional drag to the turbulent spectrum, and is sensitive to the dimensionality of the flow through the dependence of the turbulent spectrum on the dimensionality. Our findings lead 
a
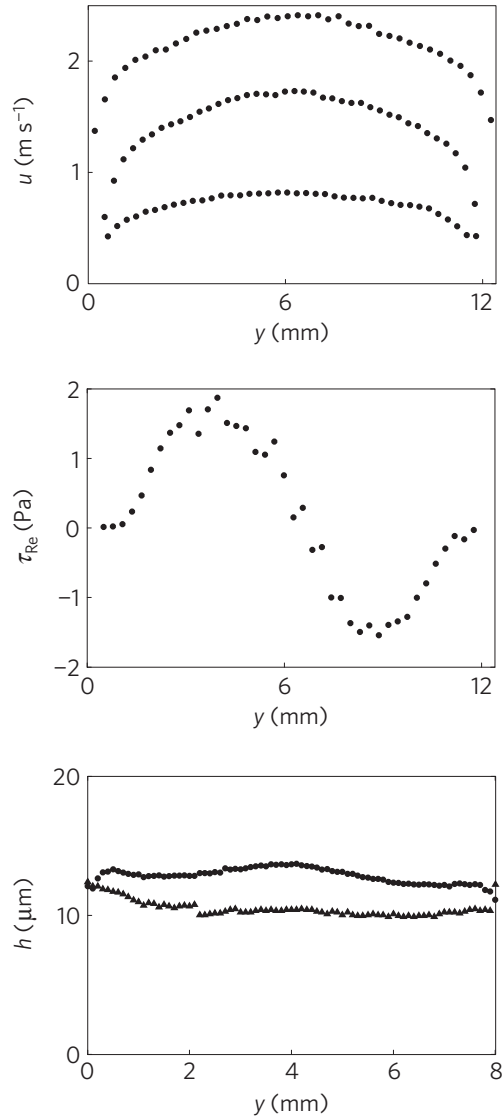

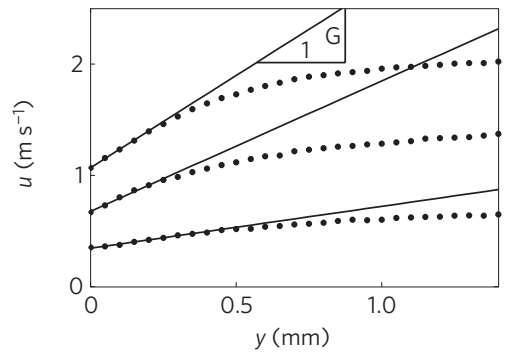

d
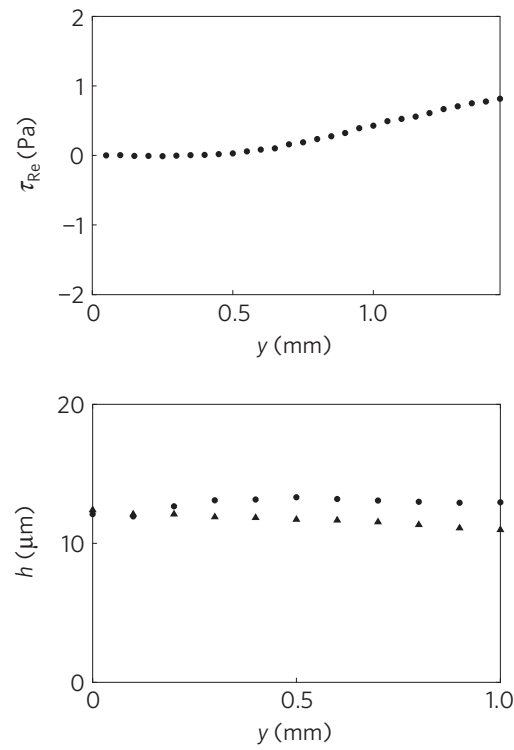

Figure 2 | The mean velocity profile $u(y)$, the Reynolds shear stress profile $\boldsymbol{\tau}_{\mathbf{R e}}(\boldsymbol{y})$ and the thickness profile $\boldsymbol{h}(\boldsymbol{y})$ in turbulent soap-film flows. a, Typical plots of $u(y)$ in a film of width $w=12 \mathrm{~mm}$, for $\operatorname{Re}=7,890,17,600$ and 25,900. From LDV measurements (see the Methods section). $\mathbf{b}$, The same as in $\mathbf{a}$ but close to one of the wires. In the viscous layer the slope of $u(y)$ is constant, $d u(y) / d y=G$. Points on the film closer than $\approx 20 \mu m$ (the diameter of the beam of the LDV) to the edge of the wire cannot be probed with the LDV; thus, the first data point, which we position at $y=0$, is at a distance of $\approx 20 \mu m$ from the edge of the wire. c, Plot of $\tau_{\text {Re }}(y)$ in a film of width $w=12 \mathrm{~mm}$, for Re $=17,600$. From LDV measurements (see the Methods section). $\mathbf{d}$, The same as in $\mathbf{c}$ but close to one of the wires. The Reynolds shear stress vanishes in the viscous layer (compare with the plots in $\mathbf{b}$ ). $\mathbf{e}$, Typical plots of $h$ ( $y$ ) in a film of width $w=8 \mathrm{~mm}$, for $\operatorname{Re}=9,430$ and 13,000. From fluorescent-dye measurements (see the Methods section). $\mathbf{f}$, The same as in $\mathbf{e}$ but close to one of the wires. The thickness of the film is nearly uniform in the viscous layer (compare with the plots in b).

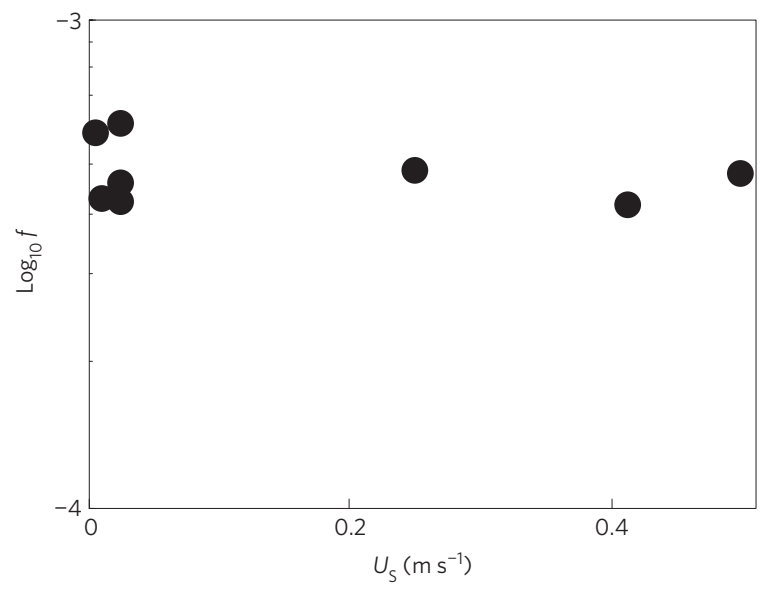

Figure 3 | Log-linear plot of the frictional drag $f$ versus the apparent slip velocity $U_{S}$ in 2D turbulent soap-film flows of Reynolds number $\operatorname{Re}=20,000$.

us to conclude that the macroscopic properties of both $3 \mathrm{D}$ and 2D turbulent flows are closely linked to the turbulent fluctuations. In addition, our findings serve to underscore the value of using $2 \mathrm{D}$ soap-film flows to test and extend our understanding of turbulence.

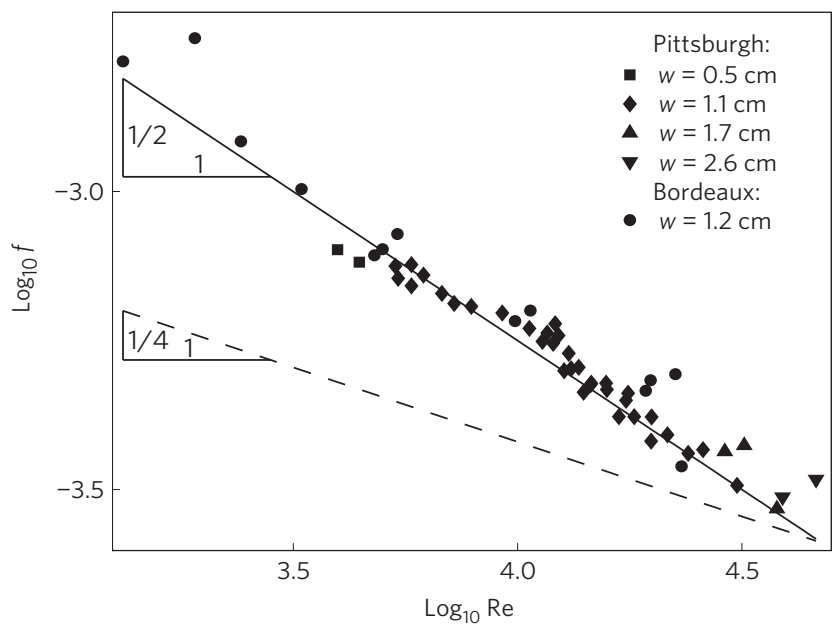

Figure 4 | Log-log plot of the frictional drag $f$ versus the Reynolds number Re in 2D turbulent soap-film flows of Reynolds number $1,300 \leq R e \leq 46,000$, from independent experiments carried out in Pittsburgh and Bordeaux. The cloud of data points may be represented as a straight line of slope $1 / 2$, consistent with the scaling $f \propto \operatorname{Re}^{-1 / 2}$. The straight dashed line of slope $1 / 4$ corresponds to the Blasius empirical scaling, $f \propto \operatorname{Re}^{-1 / 4}$. 


\section{Methods}

To measure the components $u(t)$ (along the mean flow) and $v(t)$ (transverse to the mean flow) of the instantaneous velocity at a point on the film, we use an LDV with a sampling rate of $5 \mathrm{kHz}$. By carrying out measurements over a time period of about $200 \mathrm{~s}$, we collect time series $u\left(t_{i}\right)$ and $v\left(t_{i}\right)$. From these time series we calculate the local mean velocities $u$ and $v$ as the time averages, $u \equiv\left\langle u\left(t_{i}\right)\right\rangle$ and $v \equiv\left\langle v\left(t_{i}\right)\right\rangle$. To compute the local turbulent spectrum (more precisely, the longitudinal turbulent spectrum), we invoke Taylor's frozen-turbulence hypothesis ${ }^{6}$ to carry out a space-for-time substitution $t \rightarrow x / u$ on the time series $\left(u\left(t_{i}\right)-u\right)$. This space-for-time substitution gives the space series, $u^{\prime}\left(x_{i}\right) \equiv\left(u\left(x_{i} / u\right)-u\right)$, where $x_{i}=u t_{i}$. (The frozen-turbulence hypothesis is justified because in all our experiments the root mean square of the velocity fluctuations is less than $20 \%$ of $u$ (ref. 30).) The spectrum $E(k)$ is the square of the magnitude of the discrete Fourier transform of $u^{\prime}\left(x_{i}\right)$. We compute the local Reynolds shear stress as $\tau_{\operatorname{Re}}=\rho\left\langle\left(u\left(t_{i}\right)-u\right)\left(v\left(t_{i}\right)-v\right)\right\rangle$. To measure the thickness $h$ of the film, we mix the soapy solution with a fluorescent dye and focus a blue laser beam of diameter $20 \mu \mathrm{m}$ on a spot of the film. The spot becomes fluorescent, and we monitor the intensity of the fluorescence by means of a photodetector with a counting rate proportional to $h$.

Received 25 August 2009; accepted 19 April 2010; published online 23 May 2010

\section{References}

1. Frisch, U. Turbulence: The Legacy of A. N. Kolmogorov (Cambridge Univ. Press, 1995).

2. Sreenivasan, K. R. Fluid turbulence. Rev. Mod. Phys. 71, S383-S395 (1999).

3. Pope, S. B. Turbulent Flows (Cambridge Univ. Press, 2000).

4. Hof, B., Westerweel, J., Schneider, T. M. \& Eckhardt, B. Finite lifetime of turbulence in shear flows. Nature 443, 59-62 (2006).

5. Schlichting, H. \& Gersten, K. Boundary-Layer Theory (Springer, 2000)

6. Taylor, G. I. The spectrum of turbulence. Proc. R. Soc. Lond. A 164, 476-490 (1938).

7. Sreenivasan, K. R. The phenomenology of small-scale turbulence. Annu. Rev. Fluid Mech. 29, 435-472 (1997).

8. Kellay, H. \& Goldburg, W. I. Two-dimensional turbulence: A review of some recent experiments. Rep. Prog. Phys. 65, 845-894 (2002).

9. Kraichnan, R. H. Inertial ranges in two-dimensional turbulence. Phys. Fluids 10, 1417-1423 (1967).

10. Batchelor, G. K. The Theory of Homogeneous Turbulence (Cambridge Univ. Press, 1953).

11. Richardson, L. F. Atmospheric diffusion shown on a distance-neighbour graph. Proc. R. Soc. Lond. A 110, 709-737 (1926).

12. Kolmogorov, A. N. Local structure of turbulence in incompressible fluid at a very high Reynolds number. Dokl. Akad. Nauk. SSSR 30, 299-302 (1941) (English translation in Proc. R. Soc. Lond. Ser. A 434; 1991).

13. Mckeon, B. J., Zagarola, M. V. \& Smits, A. J. A new friction factor relationship for fully developed pipe flow. J. Fluid Mech. 538, 429-443 (2005).

14. Gioia, G. \& Bombardelli, F. A. Scaling and similarity in rough channel flows. Phys. Rev. Lett. 88, 014501 (2002).

15. Gioia, G. \& Chakraborty, P. Turbulent friction in rough pipes and the energy spectrum of the phenomenological theory. Phys. Rev. Lett. 96, 044502 (2006)
16. Guttenberg, N. \& Goldenfeld, N. Friction factor of two-dimensional rough-boundary turbulent soap film flows. Phys. Rev. E 79, 065306 (2009).

17. Nikuradze, J. Stromungsgesetze in rauhen Rohren. VDI Forschungsheft 361 (1933). (English translation available as National Advisory Committee for Aeronautics, Tech. Memo. 1292 (1950). Available at http://hdl.handle.net/2060/19930093938).

18. Jiménez, J. Turbulent flows over rough walls. Annu. Rev. Fluid Mech. 36, 173-196 (2004).

19. Chézy, A. Memoire sur la vitesse de l'eau conduit dans une rigole donne. In Dossier 847 (MS 1915)(Ecole des Ponts et Chaussees, 1775). (English translation in Journal, Association of Engineering Societies 18, 363-368; 1897).

20. Dooge, J. C. I. in Channel Flow Resistance: Centennial of Manning's Formula 136-185 (ed. Yen, B. C.) (Water Resources Publications, 1992).

21. Reynolds, O. An experimental investigation of the circumstances which determine whether the motion of water shall be direct or sinuous, and of the law of resistance in parallel channels. Phil. Trans. R. Soc. Lond. 174, 935-982 (1883).

22. Allen, J. J., Shockling, M. A., Kunkel, G. J. \& Smits, A. J. Turbulent flow in smooth and rough pipes. Phil. Trans. R. Soc. A 365, 699-714 (2007).

23. Barenblatt, G. I. Scaling laws for fully developed turbulent shear flows. Part 1. Basic hypotheses and analysis. J. Fluid Mech. 248, 513-520 (1993).

24. Barenblatt, G. I. \& Chorin, A. J. A mathematical model for the scaling of turbulence. Proc. Natl Acad. Sci. USA 101, 15023-15026 (2004).

25. Kellay, H., Wu, X. L. \& Goldburg, W. I. Experiments with turbulent soap films. Phys. Rev. Lett. 74, 3975-3978 (1995).

26. Gioia, G., Chakraborty, P. \& Bombardelli, F. A. Rough-pipe flows and the existence of fully developed turbulence. Phys. Fluids 18, 038107 (2006).

27. Goldenfeld, N. Roughness-induced critical phenomena in a turbulent flow. Phys. Rev. Lett. 96, 044503 (2006).

28. Calzetta, E. Friction factor for turbulent flow in rough pipes from Heisenberg's closure hypothesis. Phys. Rev. E 79, 056311 (2009).

29. Mehrafarin, M. \& Pourtolami, N. Intermittency and rough-pipe turbulence. Phys. Rev. E 77, 055304 (2008).

30. Belmonte, A., Martin, B. \& Goldburg, W. I. Experimental study of Taylor's hypothesis in a turbulent soap film. Phys. Fluids 12, 835-845 (2000).

\section{Acknowledgements}

We have benefited from discussions with J. M. Larkin. This work was financially supported by the US National Science Foundation through NSF/DMR grant 06-04477 and NSF/DMR grant 06-04435 (W. Fuller-Mora, Programme Director). T.T. acknowledges support from the Vietnam Education Foundation. P.C. acknowledges support from the Roscoe G. Jackson II Research Fellowship.

\section{Author contributions}

Experiments were carried out primarily in Pittsburgh by T.T., and in Bordeaux by H.K. Analysis of data was carried out by all authors. Research was designed by G.G., N. Goldenfeld and W.G. G.G. wrote the paper with assistance from N. Goldenfeld and P.C.

\section{Additional information}

The authors declare no competing financial interests. Reprints and permissions information is available online at http://npg.nature.com/reprintsandpermissions. Correspondence and requests for materials should be addressed to P.C. 